\title{
Gesellschaftliche Kosten und individuelle Wirkungen
}

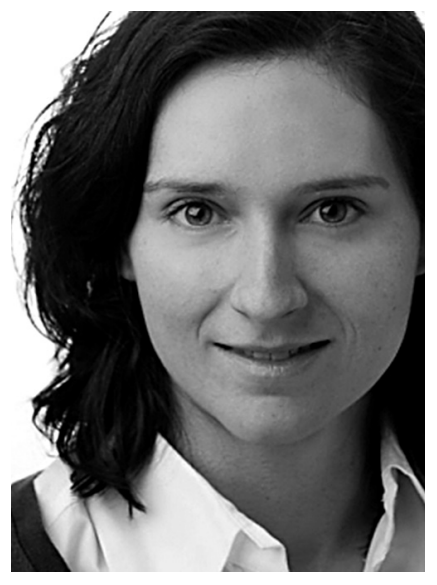

VON BRITTA WAGNER

Dr. Britta Wagner ist Soziologin und Diplom-Pädagogin. Sie lehrte an der Universität Bamberg Methoden der empirischen Sozialforschung und ist seit 2006 Beraterin bei xit forschen. planen. beraten. Das deutschlandweit agierende Beratungsunternehmen ist auf die Sozialwirtschaft spezialisiert. Sie bearbeitet dort schwerpunktmäßig die Bereiche Social Return on Investment/ Wirkung und ist unter anderem für zahlreiche Projekte in der beruflichen Rehabilitation und Behindertenhilfe verantwortlich. wagner@xit-online.de

\author{
Das Konzept des Social Return on Investment \\ (SROI) versucht eine Abkehr von nur am Input \\ orientierten Ansätzen bei der Betrachtung sozialer \\ Maßnahmen. Einbezogen werden bei diesem \\ differenzierten Rechenmodell sowohl der durch \\ soziale Dienste und Einrichtungen geschaffenen \\ gesellschaftlichen Mehrwert als auch die Wirkungen \\ bei den verschiedenen Nutzergruppen.
}

Die Steigerung der Ausgaben im Bereich der Eingliederungshilfe ist allfällig bekannt, auch deren Gründe sind weitgehend beschrieben: geringerer Altersdurchschnitt in der Klientel als in der Gesamtbevölkerung, biografisch früherer Eintritt in Hilfen, verbesserte medizinische Versorgung und bessere Lebensbedingungen. (1)

Bei einem Gesamtvolumen von knapp 13 Millionen Euro, das entspricht $57 \%$ der gesamten SGB-XII-Ausgaben (2), ist es daher nicht verwunderlich, dass die Eingliederungshilfe stark in den Fokus der Finanzpolitik gerät. Und es ist keine Überraschung, dass im horizontal wie vertikal gegliederten System der Finanzierung sozialer Dienstleistungen das Denken in Finanzierungstöpfen einen aus fachlicher und gesellschaftspolitischer Sicht überfälligen Diskurs angestoßen hat.

Seit über fünf Jahren arbeitet die Arbeits- und Sozialministerkonferenz an der Weiterentwicklung der Eingliederungshilfe für Menschen mit Behinderungen " und hat ihr 2010 vorgelegtes Eckpunktepapier »für die Reformgesetzgebung Weiterentwicklung der Eingliederungshilfe für Menschen mit Behinderungen « Mitte 2012 konkretisiert. (3) Politisch brisant sind die Überlegungen vor allem wegen der darin enthaltenen Ankündigung, in der kommenden Legislaturperiode ein Bundesleistungsgesetz zu erarbeiten. Dieses soll im Rahmen der neuen Vorgaben des Fiskalpaktes zur Entlastung der Kommunen bei der Eingliederungshilfe führen. (4)

Die aktuelle finanzierungspolitische Diskussion hat zwei grundlegende $\mathrm{Fa}$ cetten: Wer soll künftig Eingliederungshilfe finanzieren und wie soll das geschehen? Dieser Artikel beleuchtet beide Facetten hinsichtlich möglicher Konsequenzen für Werkstätten für behinderte Menschen und zeigt auf, welches Potenzial Analysen des Social Return on Investment (SROI) für diesen Diskurs haben können.

\section{Die fünf Stufen des Social Return on Investment}

Der in Kooperation von xit $\mathrm{GmbH}$, der Katholischen Universität Eichstätt und der Evangelischen Hochschule Nürnberg entwickelte Ansatz des Social Return on Investment (5) macht zum einen Mittelströme in der Finanzierung und Wirkung sozialer Dienstleistungen sichtbar und ermöglicht so die Bestimmung von gesellschaftlichen Nettokosten (SROI 1, SROI 2). Zum anderen fragt dieser Ansatz nach Kosten und Erträgen, die durch alternative Settings entstünden und ermöglicht so Allokationsvergleiche (SROI 3).

Mit Hilfe des Social Return on Investment lassen sich aber nicht nur Wir- 


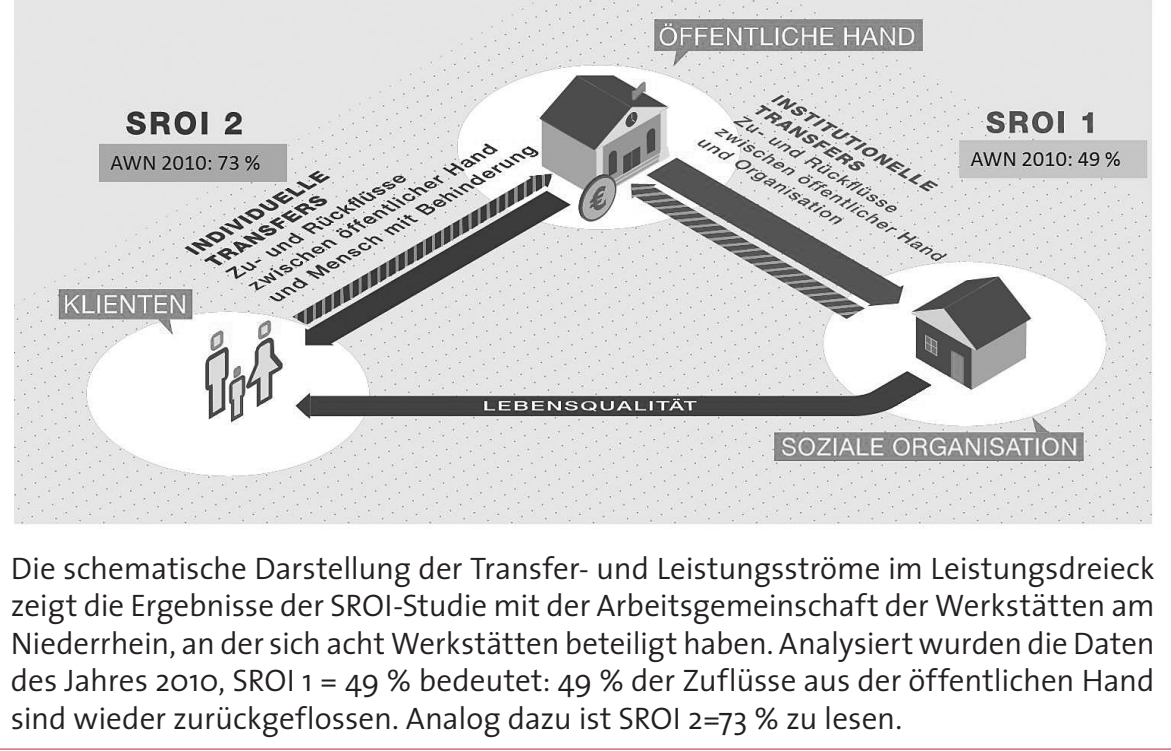

kungen darstellen, die durch monetäre Transfers an Leistungserbringer oder Leistungsnehmer entstehen. Auch die erheblichen Effekte einer Werkstatt für behinderte Menschen für eine Wirtschaftsregion lassen sich abbilden (SROI 4). Seit kurzem liegt zudem auch ein Instrument zur Messung der Lebensqualität von Kunden sozialer Dienstleistungen vor, das im Bereich der Werkstätten derzeit im Pilotprojekt eingesetzt wird (SROI 5).

Auf diese Weise lässt sich der nach unserem sozialstaatlichen Prinzip komplexe Investitions- und Wirkungskreislauf nun ganzheitlich analysieren und beispielsweise in Form einer Sozialbilanz kompakt ausweisen.

\section{Wer die Werkstatt für behinderte Menschen finanziert}

Die Frage, woher die Mittel kommen, mit denen die öffentliche Hand Werkstätten finanziert und Werkstattmitarbeiter unterstützt, konnten wir bereits mehrfach in konkreten Social-Returnon-Investment-Projekten mit Werkstätten für behinderte Menschen aus verschiedenen Bundesländern differenziert analysieren.

Die gute Nachricht zuerst: Von jedem Euro, den die öffentliche Hand für Werkstätten ausgibt, erhält sie zwischen 49 und 53 Cent zurück.

Ist die Eingliederungshilfe also im Grunde nur »halb so teuer«? Die Antwort lautet: Jein! Unsere Analysen zei- gen, dass in den meisten Ländern vor allem kommunale Mittel (bis zu $64 \%$ der Zuflüsse) in die Werkstattarbeit fließen, aber über Sozialversicherungsbeiträge und Steuereinnahmen der Bund und die Sozialkassen zwischen $82 \%$ $(18 \%+65 \%)$ und $90 \%(6 \%+83 \%)$ der Rückflüsse erhalten.

Dies hat den Landschaftsverband Westfalen Lippe (LWL) veranlasst, die Mittelströme der gesamten Eingliederungshilfe in Nordrhein-Westfalen nach dieser Social-Return-on-InvestmentLogik genauer untersuchen zu lassen (exakte Ergebnisse liegen noch nicht vor), um mit Zahlenmaterial in die Diskussion um das Bundesleistungsgesetz treten zu können. Wenn das Gesetz dem Prinzip des finanziellen Ausgleichs und der Konnexität folgt, wäre eine höhere Beteiligung des Bundes an der Finanzierung der Eingliederungshilfe notwendig.

Neben dieser bislang nicht berücksichtigen Facette einer Umverteilung kommunaler Mittel auf die Ebene des Bundes bzw. der Parafisci, führt Schütte (6) noch weitere Gründe an, die für eine Übernahme der finanziellen Verantwortung in der Eingliederungshilfe durch den Bund sprechen:Zum Ersten würde sich durch ein Bundesleistungsgesetz die Leistungsgewährung von der Nachrangigkeit im Sinne einer einkommensabhängigen Ausfallbürgschaft auch rechtlich zu dem entwickeln, was sie in der Regel ist: zum »erst- und letztzuständliche[n] Leistungssystem« (7).
- Zum Zweiten verspricht sich Schütte davon mehr Gleichheit in der Leistungsgewährung durch zentrale und stabilere Finanzierung. Er vergleicht die regional unterschiedliche Ausgestaltung mit einem Lotteriespiel, bei dem der Zugang zu Hilfen mit Rechtsanspruch durchaus vom Wohnort abhängen kann.

- Zum Dritten moniert Schütte, das institutionalisierte Leistungserbringungsrecht führe derzeit zu einer unguten Dynamik aus Monopolnachfrage und Anbieterkonkurrenz, in der die Machtposition der Leistungsträger das Gefüge zu einem Pseudomarkt mache - mit der Folge von Dumping zulasten der Angebotsqualität und damit der Leistungsnehmer.

Doch wie hier ein Leistungsgesetz Abhilfe schaffen soll, bleibt unklar. Der Vorschlag der Arbeits- und Sozialministerkonferenz verschärft dieses Problem möglicherweise noch. Künftig sollen demnach die Leistungsnehmer neben der Werkstatt für behinderte Menschen auch Leistungsmodule von anderen Anbietern wählen können. Dies soll mit einer stärkeren Personenzentrierung in der Eingliederungshilfe verbunden sein, bei der die anbieterunabhängige Bedarfsfeststellung und individuelle Leistungsangebote forciert werden sollen. Personenzentrierte Systeme werden dabei einerseits für Kosteneinsparungen für die öffentliche Hand durch den Abbau von Überversorgung bei Einzelnen sorgen. Sie werden auf der anderen Seite durch die Kosten der personenzentrierten Steuerung wieder steigen.

Insbesondere aber die zahlreichen Nebeneffekte sind zu bedenken: Wie wird das "System Werkstatt « funktionieren und wie teuer wird es, wenn die Leistungsstärkeren fehlen - ohne in den ersten Arbeitsmarkt vermittelt zu sein? Welche Kosten der Unterauslastung werden entstehen, wenn die Werkstatt einerseits einen sozialunternehmerischen Betrieb aufrechterhalten muss, dann aber jederzeit damit rechnen muss, abgewählt $\mathrm{zu}$ werden?

Solange aber nur die Werkstatt für behinderte Menschen alle Module anbieten muss, die Konkurrenten aber nicht, besteht die Gefahr des Rosinenpickens (»cream skimming «), das aus anderen Branchen der Sozialwirtschaft bekannt ist: Maßnahmen für diejenigen, 
die in der Werkstatt als Leistungsträger fungieren, können von Einzelanbietern günstig bereitgestellt werden, weil vergleichsweise wenig Betreuungsaufwand besteht. Die Qualität der in den Werkstätten zu bearbeitenden Aufträge würde zwangsläufig sinken - es sei denn, man hielte mit dem Einsatz von Produktionshelfern dagegen.

Für die Werkstattbetriebe würde der Anreiz weiter sinken, Aufträge zu akquirieren, die aus Teilhabesicht hochwertig sind, weil sie ein breites Spektrum von Aufgaben verschiedener Anspruchsniveaus bei vergleichsweise hohem Lohn- beits- und Sozialministerkonferenz in ihrem Grundsatzpapier bei der Zulassung weiterer Anbieter neben den Werkstätten ebenfalls aufgreift: »Leistungsmodule sollen Menschen mit Behinderungen in die Lage versetzen, wahlweise auch Leistungen unterschiedlicher Leistungsanbieter in Kombination in Anspruch nehmen zu können.« (8)

Eine Errungenschaft ist das vorgesehene Rückkehrrecht in die Werkstatt für behinderte Menschen. Doch noch ist unklar, ob die Erstattung der Rentenversicherungsbeiträge durch den Bund in Höhe der Differenz zu achtzig Pro-

\section{"Von jedem Euro, den die öffentliche Hand}

\section{für Behindertenwerkstätten ausgibt, erhält sie zwischen 49 und 53 Cent zurück"}

niveau beinhalten. Die Lebensqualität derer, die in den Werkstätten »zurückgelassen « würden (und das wäre die Mehrzahl), würde gemessen am Anregungspotenzial und der Heterogenität des Arbeitsumfeldes deutlich sinken.

Im ungünstigsten Fall entstehen sowohl Mehrkosten, die nicht durch die Sozialunternehmen zu schultern sein werden, sondern vom Sozialleistungsträger, als auch für eine große Zahl von Leistungsnehmern eine Angebotsverschlechterung.

\section{Wie die Mittel verwendet werden sollen}

An dieser Stelle verlassen wir die Perspektive der finanzierungspolitischen Debatte und wenden uns der allokativen Wirkung der eingesetzten öffentlichen Mittel zu (unabhängig davon, aus welchem Budget diese Mittel kommen). Die entscheidende Frage lautet dabei: Wie werden die öffentlichen Mittel am besten verwendet?

Bei der Frage nach der besten Verwendung der Mittel kommen zum einen die politischen und rechtlichen Rahmenbedingungen ins Spiel. Am wichtigsten ist aber der Blick auf die erzielten Wirkungen der alternativen Settings für den Leistungsnehmer.

Eine Forderung der UN-Behindertenrechtskonvention ist das Wunsch- und Wahlrecht der Betroffenen, das die Ar- kommen (1.092 Euro), wie es beispielsweise die LAG Werkstatt für behinderte Menschen Niedersachsen im Gespräch mit der SPD Landesgruppe Niedersachsen im November 2012 im Bundestag forderte. (9) Mit dem Modell der langfristigen Entwicklung der individuellen Perspektive des Social Return on Investment ließe sich dieses leistungsträgerfinanzierte Arbeitsentgelt auf Vor- und Nachteile für die öffentlichen Hände und das Lebenseinkommen des Leistungsnehmers hin untersuchen.

Die Suche nach der optimalen Mittelallokation dreht sich letztlich um ein Preis-Leistungs-Verhältnis. Versteht man die geforderte Inklusion als gelingende Individualität (10), die in modernen Gesellschaften selbstverständlich auch innerhalb institutionalisierter Angebotsformen geschieht, dann braucht es Klarheit über zwei Dimensionen: Was kosten verschiedene Alternativen (netto) für die Gesellschaft und welche Alternative erzielt beim Leistungsberechtigten die »beste« Wirkung, gemessen am Ausmaß der durch das Angebot produzierten Lebensqualität?

Beide Fragen beantworten das Konzept des Social Return on Investment in der Alternativenbetrachtung und der Lebensqualität. Am Beispiel der Arbeitsgemeinschaft der Werkstätten am Niederrhein zeigte sich für das Jahr 2010, dass die Werkstatt pro Platz und Jahr netto für die Gesellschaft Kosten in Höhe von gut 10.700 Euro verursacht. Realistische Alternativen, wie beispiels-

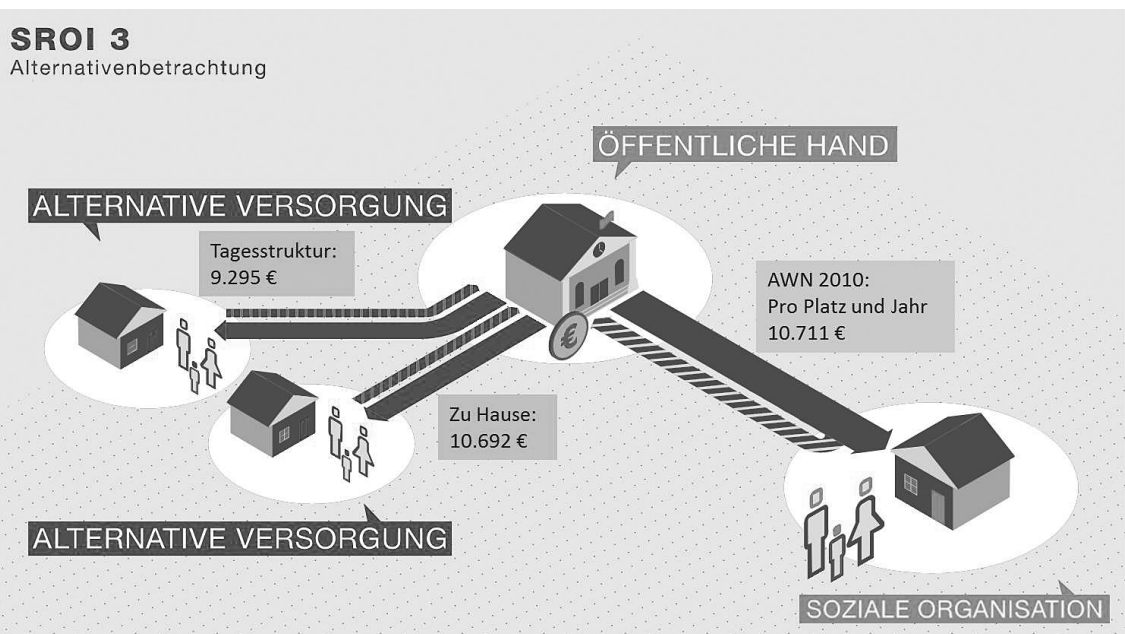

Die schematische Darstellung zeigt eine mögliche Alternativbetrachtung: Auch hier sind die Werte der SROI-Analysen der Arbeitsgemeinschaft der Werkstätten am Niederrhein aus dem Jahr 2010 eingetragen. Der Folgeschritt wäre hier, die Alternativen hinsichtlich ihrer Lebensqualitätswirkung zu bewerten und zu einer Art Preis-Leistungs-Verhältnis zu kommen. 
weise die Tagesstruktur oder der Verbleib als Arbeitsloser zu Hause wären kaum günstiger, weil sich beispielsweise höhere Betreuungsschlüssel in der Wohngruppe und der Verdienstausfall - und damit weniger Steuern und Sozialversicherungsbeiträge - von Familienangehörigen zu Buche schlagen. Die Beschäftigung in einem Integrationsbetrieb wäre für die öffentliche Hand deutlich günstiger, aber nur in den Fällen, in denen die Eigenproduktivität der Mitarbeiter Löhne erlaubt, die deutlich über dem vorgeschlagenen Grundeinkommen von 1.092 Euro liegen.

Nimmt man nun die Wirkung der verschiedenen Alternativen auf die Lebensqualität der Betroffenen hinzu, wird deutlich, zu welchen (Netto-) Kosten welches Maß an Lebensqualität produziert wird. Die Messungen sind derzeit noch im Pilotstadium, aber erste Ergebnisse zeigen bereits, wie individuell Lebensqualitätsdimensionen ausgeprägt sein können und geben Hinweise darauf, wie Angebotsformen darauf reagieren sollten.

\section{Resümee}

Über den Analyseansatz des Social Return on Investment ließen sich bei einem breiten Spektrum von sozialen Dienstleistungsangeboten - wie beispielhaft an der Institution der Werkstatt für behinderte Menschen gezeigt wurde gesellschaftliche Kosten und individuelle Wirkungen in einer ganzheitlichen Sicht zusammenbringen. So ist es auch möglich, verschiedene Finanzierungsmodelle kurz- und langfristig zu simulieren und mittels Stakeholder-Analysen finanzielle Vor- und Nachteile sowie funktionale und dysfunktionale Anreize zu identifizieren.

\section{Anmerkungen}

(1) Vgl. u. a. Schütte, W. (2012): Abschied von der »Eingliederungshilfe«. Ein Leistungsgesetz zur sozialen Teilhabe für Menschen mit Behinderungen? Teil 1. In: Nachrichtendienst des Deutschen Vereins für Öffentliche und Private Fürsorge (NDV). Dezember 2012. S. 575-585.

(2) Vgl. BAGüS (Hg.) (2012): Kennzahlenvergleich der überörtlichen Träger der Sozialhilfe 2011. Online verfügbar unter www.lwl.org/ spur-download/bag/kennzahlenvergleich2011.ppdf. Letzter Abruf: 14.04.13.

(3) ASMK (2012): Grundlagenpapier zu den Überlegungen der BundLänder-Arbeitsgruppe »Weiterentwicklung der Eingliederungshilfe für Menschen mit Behinderungen“ der ASMK. 23. August 2012. Online verfügbar unter www.lwl.org/ spur-download/bag/52_12an1.pdf. Letzter Abruf: 14.04.13.

(4) Vgl. Bundesdrucksache 282/12.

(5) Vgl. Schellberg, K. (2010): Mehrwert oder Kostenfaktor? Der Social Return on Investment in Sozialunternehmen. In: Werkstatt:Dialog, Nr. 6.2010. S. 44-47. Und: Wagner, B., Halfar, B. (2011): Soziales wirkt. Teil 1: Der Social Return on Investment bewährt sich in der Praxis. In: Bank für Sozialwirtschaft (Hrsg.): BFS-Info 10/11. Online verfügbar unter www.sozialbank. de/bfs_publikationen/\#c631.

(6) Schütte 2012, siehe Anmerkung 1.

(7) Ebd.:580.

(8) ASMK 2012:5, siehe Anmerkung 3.

(9) Vgl. LAG WfbM Niedersachsen (2012): Offene Gespräche mit der SPD-Landesgruppe Niedersachsen im Bundestag. Nachrichtenmeldung vom 3.12.2012. Online verfügbar unter www.lag-wfbmniedersachsen.de/?id=585. Letzter Abruf: 14.04.13.

(10) Vgl. Wagner, B.; Weber, M. (2013): Die Messung von Lebensqualität als Ansatzpunkt für ein fachliches Wirkungscontrolling in Werkstätten. Sieben Thesen, warum sich Werkstätten für behinderte Menschen der Inklusionsdiskussion stellen müssen und wie das gelingen kann. Vortrag im Rahmen der Werkstättenmesse Nürnberg am 14.3.2013.

\section{Neu in der Edition}

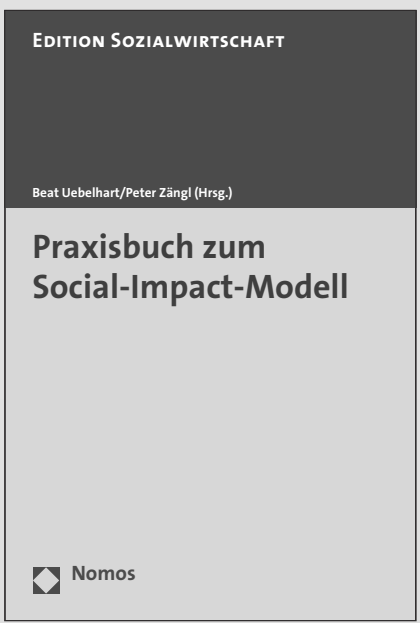

\section{Praxisbuch zum Social-Impact-Modell}

Herausgegeben von Prof. Beat Uebelhart und Prof. Dr. Peter Zängl 2013, 371 S., brosch., 49,-€ ISBN 978-3-8487-0089-9

(Edition Sozialwirtschaft, Bd. 36)

Das Praxisbuch zum SocialImpact-Modell stellt theoriegestützte Tools, Methoden und Instrumente zur Entwicklung, Planung, Steuerung und Kontrolle sozialer Hilfen bereit. Es schließt somit die Lücke zwischen Theorie und Praxis und widmet sich der Fragestellung: „Wie funktioniert das SocialImpact-Modell in der praktischen Anwendung?"

www.nomos-shop.de/20203

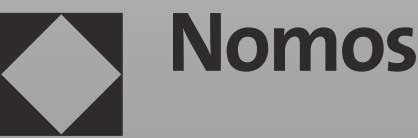

